\title{
ANÁLISE DA APLICAÇÃO DE ROADMAPPING EM AMBIENTE DIGITAL COMO APOIO À FORMULAÇÃO DE ESTRATÉGIA EM UMA UNIVERSIDADE
}

Carolina Campos Nagem Araújo (ㅁagemcarolina@gmail.com) - Pontifícia Universidade Católica de Minas Gerais, PUC-Minas.

Gabriel Pereira Soares Barbosa (voltsgabriel@hotmail.com) - Pontifícia Universidade Católica de Minas Gerais, PUC-Minas.

Matheus Luiz Pontelo de Souza (mslzposa@gmail.com) - Pontifícia Universidade Católica de Minas Gerais, PUC-Minas.

\section{RESUMO}

O método de planejamento estratégico Roadmapping é utilizado mundialmente para que seja possível identificar, explorar e mapear estratégias de uma organização. Sua utilização é extensa e prática, podendo ser aplicado desde o planejamento estratégico de uma microempresa, até a elaboração de um plano governamental. No entanto, alguns casos não são amplamente difundidos na literatura, como no planejamento estratégico de uma universidade, fator que gera desafios para a aplicação do método nesses ambientes. Ademais, apesar da flexibilidade do método, sua aplicação em ambiente digital apresenta desafios especiais. Baseando-se nessas perspectivas, a pesquisa teve como base um estudo de caso com o objetivo de estudar melhores caminhos para a aplicação do Roadmapping digital no planejamento estratégico de um departamento de uma universidade. A aplicação teve o apoio de quatro integrantes de um grupo de pesquisa e o professor orientador do grupo que conduziu o projeto. Assim, a aplicação flexível do Roadmapping digital permitiu a criação de um mapa estratégico, feito digitalmente, que se mostrou útil para o objetivo dos stakeholders desse setor acadêmico. Finalmente, considerações sobre o Roadmapping em ambiente digital e sobre o potencial de abordagens contingenciais para customização do método foram expostas.

Palavras-chave: Roadmapping; SWOT; planejamento estratégico; universidade, métodos e técnicas. 


\section{INTRODUÇÃO}

O Roadmapping é um método que se consolidou mundialmente como forma de apoio ao planejamento estratégico conjunto, em empresas dos mais diversos setores. (PHAAL; FARRUKH; PROBERT, 2010). Ele se tornou útil, principalmente, na identificação e exploração de oportunidades em ambientes que tratam de tecnologia, colaborando com a gestão em ambiente tecnológico. (SOUZA; MELO FILHO; CHENG, 2020).

Um dos grandes usos do método Roadmapping é feito em empresas ou em produtos de base tecnológica. O método tem uma característica muito positiva para estes ambientes, que envolve a visualização do mapa, ou seja, da estratégia coletivamente construída, de maneira que a gestão seja feita de forma visual. Frente a um período em que todas as atividades precisavam passar para um contexto virtual, surgiu a dificuldade de adaptar a aplicação dessa ferramenta ao modelo virtual. (SOUZA et al, 2019).

Esta pesquisa surgiu da necessidade de uma Universidade privada, por meio do departamento de uma área específica, que tinha o objetivo de planejar estrategicamente os passos para a melhoria de um curso de pós-graduação. Para isso, iniciou-se a busca pela melhor maneira de adaptar esse método à presente realidade, tanto em termos de um modelo adaptado de aplicação quanto em termos da imposição da realização do Roadmapping em ambiente 100\% virtual devido à pandemia do COVID-19. No caso em estudo, os stakeholders, com o objetivo de aumentar a nota CAPES do curso, propuseram a aplicação de um método para gestão da estratégia ao longo de cinco anos, determinados por eles. Dessa forma, o processo consistiu em um modelo adaptado da aplicação do método, com o objetivo de criar um planejamento estratégico, de forma virtual, baseado em uma breve pesquisa de mercado e uma pesquisa SWOT.

O resultado desse trabalho, discutido com mais profundidade na seção 4 deste artigo, se deu com menos tempo e complexidade do que o planejado. A aplicação do Roadmapping digital em um departamento de universidade gerou resultados efetivos para o objetivo dos stakeholders, resultando em um mapa bem elaborado, na perspectiva dos clientes, com elementos visuais ricos e simples de compreender. O estudo, no entanto, rendeu considerações sobre a aplicação do método Roadmapping em ambiente digital, relacionadas à sua flexibilidade, assim como sobre a possibilidade de abordagens contingenciais para 
customização do método em diferentes aplicações, levando em conta o ambiente, os envolvidos, a complexidade do projeto, entre outros fatores.

\section{REVISÃO TEÓRICA}

\subsection{Roadmapping}

O Roadmapping é definido por Oliveira et al (2019) como uma abordagem utilizada para a identificação, definição e mapeamento das estratégias, objetivos e ações relacionados com a inovação em uma organização ou negócio. O principal resultado dessa abordagem é um mapa chamado de Roadmap e é contido nele as possíveis trajetórias estratégicas de uma organização em direção a um objetivo, as oportunidades existentes e os desafios a serem enfrentados até o destino estratégico. (OLIVEIRA et al, 2019). O Roadmap cria uma representação visual do que foi discutido no processo de Roadmapping, por isso, sua arquitetura é essencial e pode sofrer alterações dependendo da forma com que o Roadmapping é aplicado, mas tipicamente a arquitetura do Roadmap se baseia como mostrado na Figura 1. (PHAAL et al, 2004). Essa estrutura é construída ao longo de um processo baseado em oficinas, cada uma com seu objetivo, que conferem robustez ao método, fazendo com que a construção do mapa seja efetiva. Essas reuniões reúnem trabalho de diversas pessoas envolvidas estrategicamente e serão abordadas ao decorrer do estudo. (SOUZA; MELO FILHO; CHENG, 2020).

Figura 1 - Arquitetura de Roadmap comumente usada em ambiente de inovação

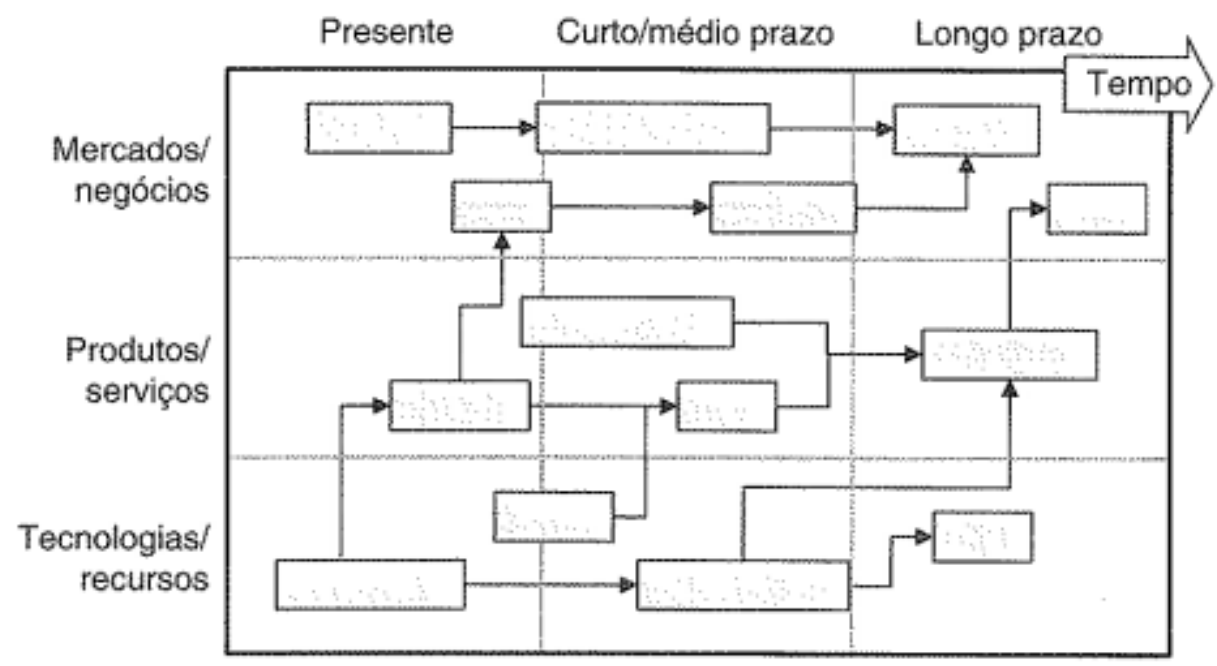

Fonte: Oliveira et al (2019) 
Segundo Oliveira et al. (2019), a utilização do Roadmapping vem crescendo nos últimos anos, fato que comprova sua ampla aceitação nas organizações. Os benefícios dessa abordagem incluem a identificação de novas oportunidades de negócios, a definição de estratégias e objetivos comuns entre diferentes organizações e o alinhamento entre estratégia comercial e tecnológica, porém não se restringem a estes, tendo em vista, segundo Phaal et al. (2020), a amplitude de aplicação do Roadmapping, podendo ser aplicada em vários casos e setores, inclusive no setor educacional.

\subsection{Aplicações do Roadmapping}

Existem formas de aplicação de Roadmapping baseadas em workshop, entre as quais T-plan e S-plan. O Roadmapping no processo de planejamento estratégico (S-Plan) tem por seu principal objetivo a exploração das possibilidades estratégicas. Sua utilização pode ocorrer em nível corporativo ou em um sistema industrial. (PHAAL; FARRUKH; PROBERT, 2007), (PHAAL; FARRUKH; PROBERT, 2010). A utilização do Roadmapping em um processo de inovação (T-Plan) tem o foco na identificação de oportunidades, geração de ideias e conceitos e no desenvolvimento de novas tecnologias e produtos e pode ser utilizado em apenas uma organização. (OLIVEIRA et al, 2019).

De acordo com Oliveira et al (2019), é muito comum a confusão que se faz o processo de Roadmapping e a construção de mapas visuais, uma vez que o método e o sucesso de sua aplicação dependem de um processo de construção e estruturação bem definido, e não apenas do Roadmap final. Por esse motivo, as abordagens T-Plan e S-Plan são apresentadas, como uma perspectiva de robustecer o processo de Roadmapping em si. (SOUZA; MELO FILHO; CHENG, 2020).

O processo de desenvolvimento do S-Plan se baseia em um workshop que se inicia em uma visão geral do contexto de inovação, e segue com a priorização dos pontos mais relevantes, até a revisão dos resultados obtidos. (PHAAL; FARRUKH; PROBERT, 2007). O processo de S-Plan possui três etapas, conforme estabelecido na Figura 2. 
Figura 2 - Visão geral do processo de Roadmapping do S-Plan

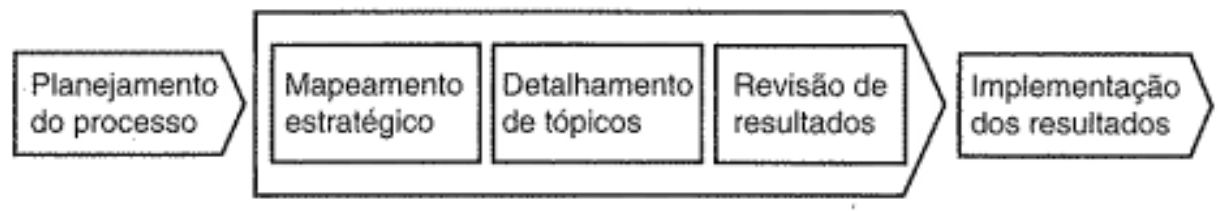

Fonte: Adaptado de Phaal (2007)

O processo do T-Plan, ilustrado na Figura 3, é iniciado com o planejamento do projeto, definindo a arquitetura do Roadmap, o escopo o processo e o que será necessário para os workshops, então são formados quatro workshops: mercado, produto, tecnologia e construção do Roadmap seguidos por uma etapa final de revisão e implementação dos resultados. (OLIVEIRA et al, 2019), (SOUZA et al, 2019).

Figura 3 - Visão geral do processo de Roadmapping do T-Plan

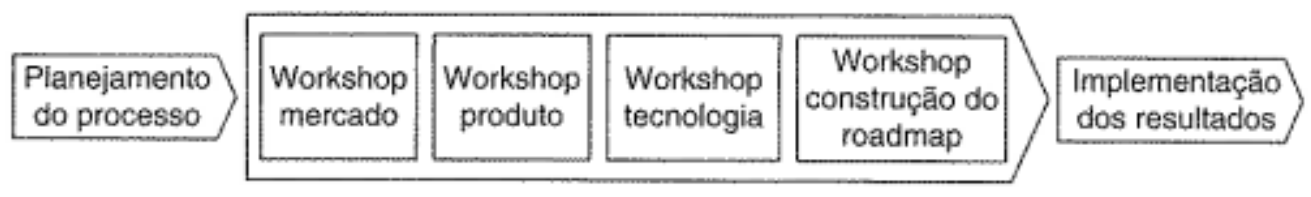

Fonte: Adaptado de Phaal et al (2001)

O T-Plan sugere diferentes usos da arquitetura do Roadmap de inovação, formada pela linha do tempo e pelas camadas, que podem ser definidas de acordo com o objetivo do mapa, mas sendo utilizadas, por exemplo, as de mercado/negócio, produto/serviço e tecnologia, que é mostrado na Figura 4. (PHAAL; FARRUKH; PROBERT, 2010). Nos workshops participam o dono do processo e os facilitadores, enquanto para a realização das etapas, é formada uma equipe de execução multifuncional. (OLIVEIRA et al, 2019).

Figura 4 - Arquitetura de Roadmap para inovação indicada pelo T-Plan 


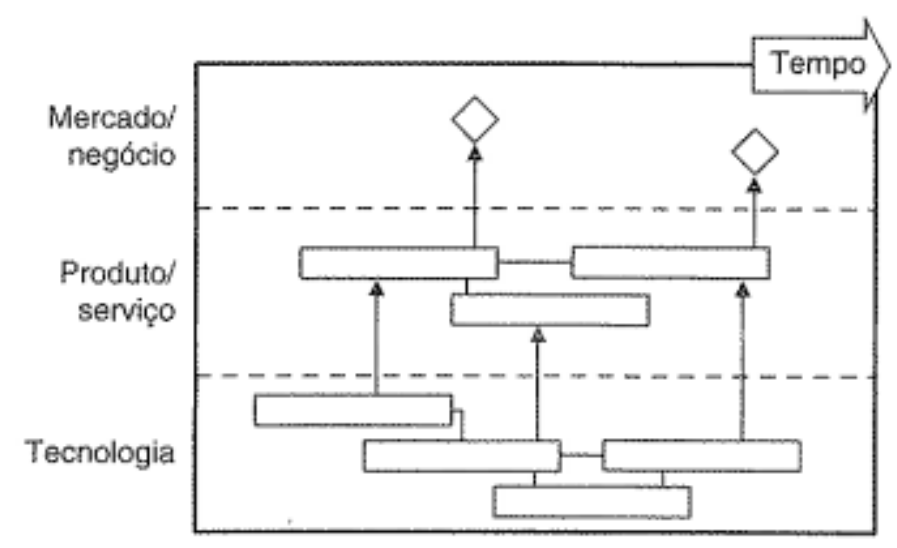

Fonte: Adaptado de Phaal et al (2001)

\subsection{O Roadmapping em ambiente digital}

Realizar apenas a abordagem "pura" do Roadmapping, ou seja, sem nenhum tipo de ferramenta que enriquece esse processo, pode gerar um resultado incompleto, que não atende todas as necessidades relacionadas com o gerenciamento e planejamento da inovação. (OLIVEIRA et al, 2019). Ademais, tendo em vista uma utilização dessa abordagem feita integralmente em ambiente digital, essas ferramentas se tornam essenciais para o sucesso do procedimento.

Com o objetivo de manter o método em seu objetivo de ser um mapa visual e flexível, colocálo em ambiente virtual exige alguns cuidados a mais. Por esse motivo, softwares de lousa digital como, por exemplo, o Mural, ajudam em uma melhor visualização e se assemelham ao que é feito em ambiente físico com post its e canetas, o que enriquece o Roadmapping, fazendo com que ele se torne mais flexível, organizado, mais fácil de visualizar e consequentemente faz com que as priorizações e análises de lacunas sejam mais precisas e simplificadas. (SOUZA et al, 2020). Além disso, há outros benefícios notáveis para a aplicação remota do Roadmapping com uso de softwares que auxiliam o procedimento, como a mobilidade do Roadmap gerado. Do modo presencial e usual de aplicação do Roadmapping, o Roadmap é feito em papel ou quadros, o que pode dificultar a mobilidade do mesmo, por exemplo para levá-lo para casa ou a uma outra sala. (SOUZA et al, 2020).

\section{METODOLOGIA}

A pesquisa foi elaborada pela aplicação do estudo de caso. O estudo de caso pode ser definido, de acordo com Yin (2005), como um conjunto de métodos que inclui, experimentos, 
estudos observacionais, levantamentos, análise de arquivos e pesquisas históricas. Desse modo, o estudo de caso pode ser formulado abrangendo diferentes métodos, como entrevistas, coleta de dados, questionários e observação participante.

O objetivo deste caso de aplicação do Roadmapping é de criar um mapa estratégico para um departamento de pós-graduação da PUC Minas, para que esse mesmo departamento pudesse no futuro alcançar uma maior nota no sistema CAPES. A aplicação desse estudo de caso se fundamentou na coleta e tratamento de dados, tendo início em agosto/2020 e finalizado em abril/2021. A aplicação teve apoio de métodos como entrevistas, observação participante, análise documental e a análise SWOT e foi conduzida a partir de oficinas, cujo objetivo era discutir sobre os dados coletados e inseri-los no estudo para se correlacionarem com o objetivo da aplicação e das partes interessadas, para a criação do Roadmap.

A aplicação foi conduzida por quatro alunos da PUC Minas integrantes do grupo de pesquisa GETI (Grupo de Empreendedorismo Tecnológico e Inovação), bem como o professor coordenador do grupo, que teve o papel de facilitador e condutor do processo. A equipe do departamento em questão, que teve o papel de cliente neste projeto, era composta por três professores-chave que, em ocasiões específicas, ativaram outros stakeholders para participar do esforço de execução do Roadmapping.

Por ser uma aplicação em meio digital, as oficinas, reuniões e entrevistas foram feitas exclusivamente em aplicativos de comunicação por videoconferência como o Google Meet e o Microsoft Teams, dos quais estiveram presentes em todas as etapas do processo. Para a viabilização da construção do Roadmap e do processo de Roadmapping, foram usados os serviços de videoconferência somados ao Mural, uma área de trabalho virtual.

\section{RESULTADOS E DISCUSSÃO}

Neste tópico, será exposta, com maior detalhamento, a forma com que o método Roadmapping foi aplicado, objetivando desenvolver um planejamento estratégico para o curso de pós-graduação de uma universidade. Paralelamente, será exposta uma análise da efetividade e adaptabilidade de aplicação do método.

\subsection{Detalhamento do processo, coleta e tratamento de dados}

Frente a uma realidade em que os stakeholders ainda não continham muito conhecimento e domínio na área da estratégia, a ideia do primeiro momento foi focar em estudos, para 
favorecer o conhecimento do processo, respondendo questões sobre mercado, produtos e tecnologia, para criação de estratégia posteriormente. Portanto, o plano inicial, definido pelas equipes envolvidas, foi de, no período de 3 meses, estudar essas três camadas, utilizando pesquisas sobre a voz do cliente e a voz do mercado, além da resposta a um questionário de 14 questões para enriquecimento do Roadmapping via análise SWOT.

Figura 5 - Processo utilizado para produção do Roadmap

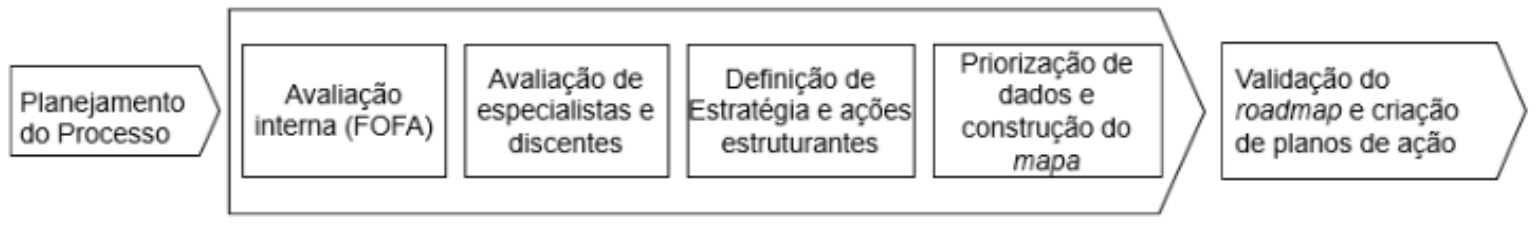

Fonte: Elaborado pelos autores

Na figura 5, está ilustrado o framework da proposta de aplicação dos métodos, resultando no Roadmap concluído e de acordo com os objetivos estabelecidos inicialmente. Iniciando com o planejamento do processo, foi nesse passo onde foi feita a definição de participantes, de técnicas auxiliares e a formalização do cronograma do processo de Roadmapping. Dessa forma, a construção de uma análise SWOT, baseada em um questionário, adaptado do modo de aplicação da SWOT proposto por Bagno e Faria (2017), foi o primeiro passo. Após a segunda e terceira etapa, que, em conjunto, captaram dados necessários para a elaboração da estratégia, a quarta e a quinta etapas, também retratadas na figura 5 , foram definidas pela escolha da ferramenta de apoio à elaboração do mapa em ambiente virtual, assim como a execução dos dois workshops.

Para a coleta de informações que fossem ajudar a preencher a análise, utilizou-se de entrevistas com os clientes - os alunos do departamento de pós-graduação em Engenharia Mecânica; e envolvimento de especialistas no contexto de programas de pós-graduação externos ao em questão. A coleta de dados foi rica para o processo de Roadmapping, sendo parâmetro para decisões estratégicas e entendimento de mercado. No entanto, a coleta de dados por meio da visão de especialistas não foi tão efetiva quanto o que se esperava, tendo em vista a objetividade da visão estratégica dos stakeholders que era clara: aumentar a nota da CAPES. Dessa forma, as maiores informações coletadas, por parte dos alunos do grupo de apoio interno, foram sobre mercado, por meio de análises de mercado e voz do cliente. 
Após obtenção das informações iniciais, para que a estratégia fosse construída de forma mais rica e abrangente, para alcançar seu objetivo é realizar um impacto positivo ao longo da sua aplicação, houve necessidade de tratar os dados coletados, pois muitas das respostas e informações encontradas apresentavam dados repetidos entre os respondentes. Foram feitas então, algumas versões de um diagrama de afinidades, utilizado tanto para a análise SWOT como para voz dos alunos, resultando em alguns pontos levantados na pesquisa que realmente poderiam ter relevância na construção dessa estratégia. $O$ resultado do diagrama de afinidades para SWOT pode ser ilustrado na Figura 6.

Figura 6 - Organização visual das categorias de respostas SWOT

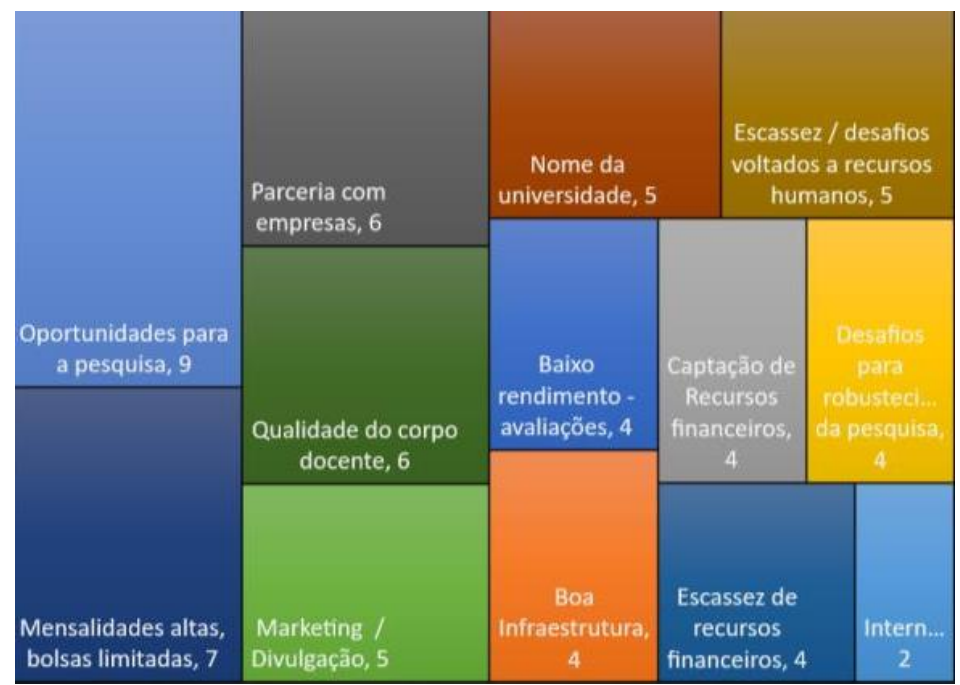

Fonte: Elaborado pelos autores

Desses pontos, surgiu a análise SWOT, clareando então, alguns aspectos que deveriam ser tratados no planejamento estratégico para os cinco anos planejados ao longo do mapa.

\subsection{O processo de Roadmapping}

Inicialmente, o processo de mapeamento e desenvolvimento de cada camada do mapa foi planejado de forma a seguir o T-Plan, sendo assim, utilizando-se de três workshops virtuais com os professores responsáveis pelo desenvolvimento do curso, com duração média de 2 a 3 horas, cada uma. O objetivo de cada workshop foi de construir, em conjunto com os stakeholders, assim como baseando-se nas pesquisas realizadas e explicadas na seção anterior, uma camada do mapa, até que se complete todas as informações necessárias e o mapa possa ser, na última reunião, estruturado em sua versão final. 
É desdobrada, inicialmente, a camada mercado, seguida da camada ações, até concluir, no penúltimo workshop, a camada recursos. Contudo, ao decorrer do processo, a data final de entrega do mapa concluído e revisado foi antecipada, por uma necessidade interna da próreitoria à qual o departamento em questão respondia.

Por esse motivo, o planejamento inicial teve que se adequar às necessidades de prazo que se alteraram, assim, foram realizadas duas reuniões abordando todos os temas e construindo o Roadmap final em paralelo, como ilustrado na Figura 7. No primeiro workshop, foi discutido sobre Metas, Ações e Recursos e foram inseridas as informações no Roadmap, de forma a criar um rascunho do mapa. Além disso, foram também feitas as ligações necessárias entre cada tarefa colocada no mapa e montando uma parte do caminho da estratégia. Já no segundo workshop, o foco foi organizar o mapa, priorizar, detalhar e, assim, chegar à versão final.

Figura 7 - Processo aplicado de construção do mapa

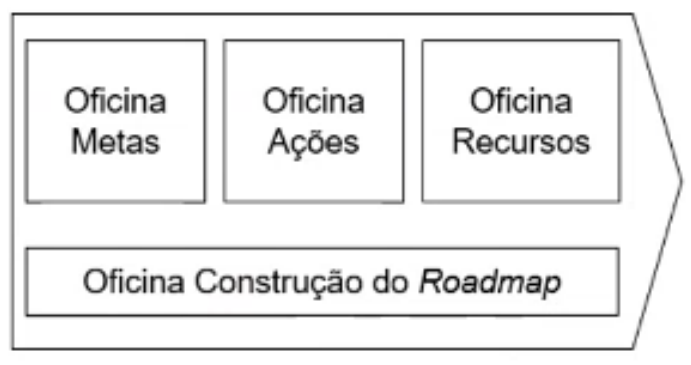

Fonte: Elaborado pelos autores

As duas reuniões tiveram duração de 2 a 3 horas e contaram com a presença do professor especialista no método Roadmapping, dos professores do departamento e de dois alunos do grupo de empreendedorismo tecnológico e inovação, para que o trabalho pudesse, futuramente, se tornar um estudo de caso.

Como resultado dessas duas reuniões, em conjunto com a análises posteriores, foi entregue, então, aos stakeholders, o Roadmap, contendo a estratégia para 5 anos de aplicação, com o objetivo que eles possuíam e com as atividades e recursos necessários para alcançar esse objetivo no período estabelecido. 


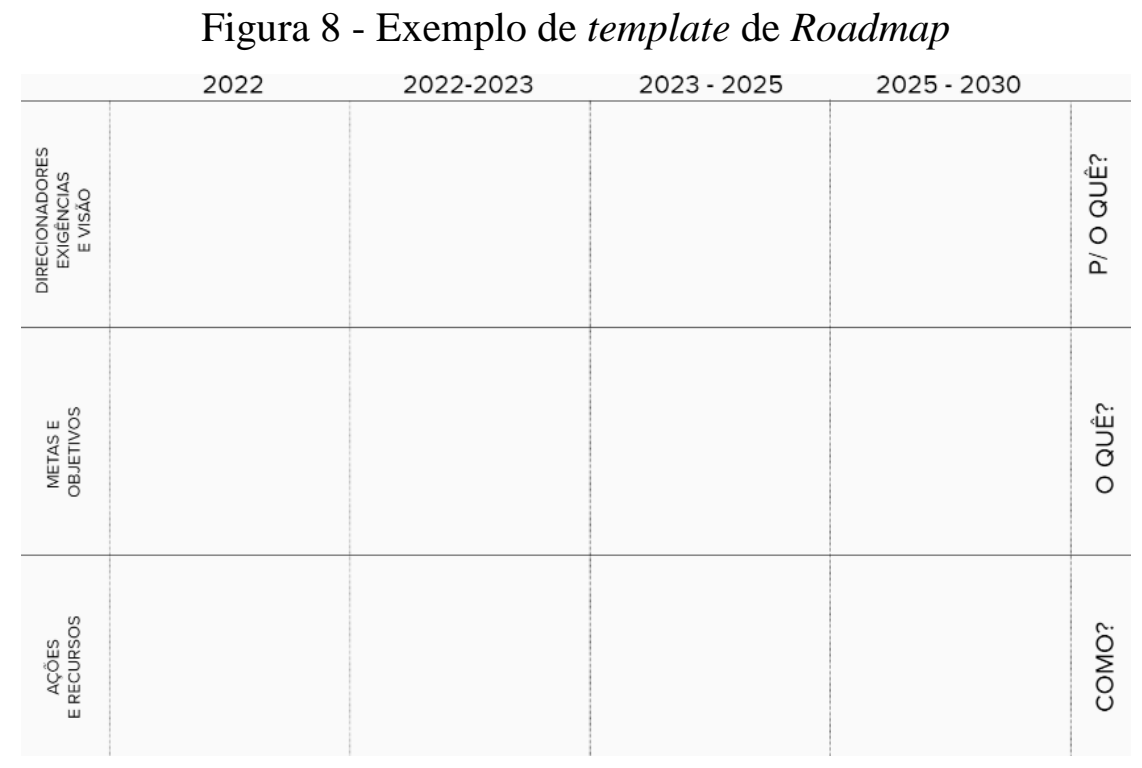

Fonte: Elaborado pelos autores

Figura 9 - Resultado do Roadmap

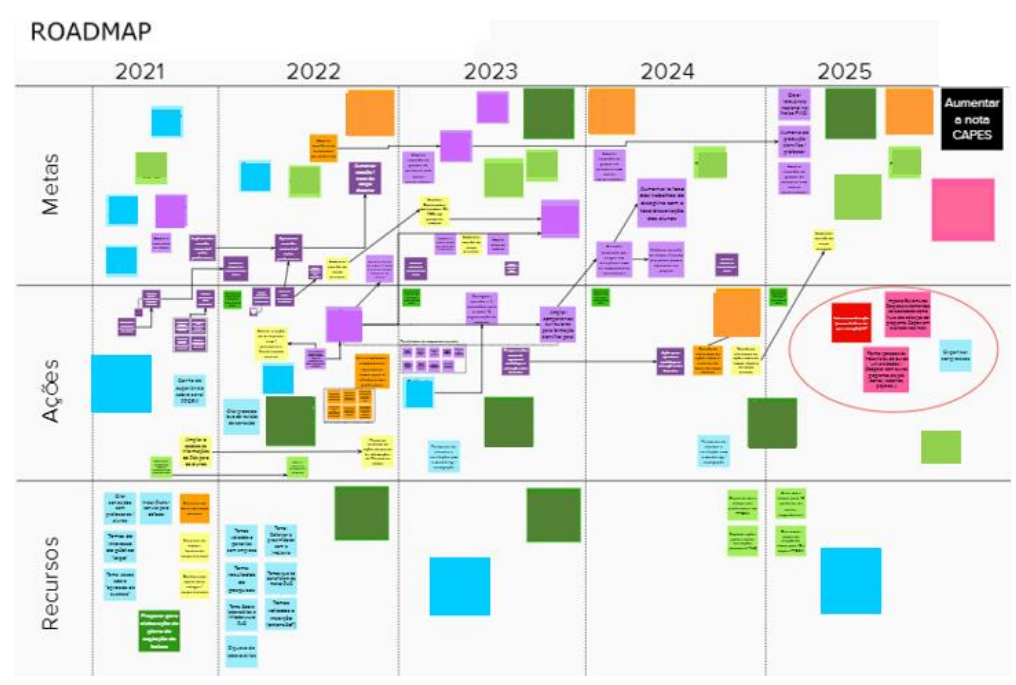

Fonte: Elaborado pelos autores

\subsection{Análise da aplicação}

O principal objetivo desse caso foi alinhar a visão dos envolvidos, para construir, tanto uma visão estratégica, como uma rota para atingi-la. Dentro desse objetivo, o resultado alcançado foi positivo e bem recebido pelos stakeholders.

Em uma análise mais aprofundada a respeito do método e sua aplicação em si, observou-se que o processo resumido não gerou prejuízos frente aos resultados esperados, levantando uma dúvida sobre a possibilidade da simplificação das lógicas de workshop/preparação, para que o 
resultado entregue seja atingido, com maior flexibilidade e agilidade. Em determinadas situações, quando é possível reduzir a informação, precisão e exigência e alcançar um resultado melhor ou igual ao que se alcançaria caso todas as etapas do método fossem seguidas à risca, pode ser uma possibilidade e até mesmo mais eficiente. Entende-se que esse caso em estudo foi assim, uma vez que o planejado inicialmente era um grande trabalho de pesquisa e levantamento de dados para a construção da estratégia do departamento e muito tempo de workshops para construção do Roadmap. E analisando, então, o proposto inicialmente para o trabalho, entendemos que as três reuniões inicialmente propostas de montagem do mapa, resumidas em duas, foram suficientes nesse caso. A utilização da lousa digital, nesse caso, do site "Mural", como apoio à montagem do mapa, foi de grande relevância, permitindo boa interação e possibilitando alterações de forma fácil e rápida.

A partir deste estudo, foi percebida uma necessidade em termos do desenvolvimento de uma abordagem contingencial para aplicação do método. No caso aqui descrito, percebeu-se que a abordagem baseada em workshops inspirada nos modelos T-Plan e S-Plan poderia ter sido sumarizada desde o início, algo percebido apenas durante o decorrer da aplicação. Em realidades de aplicação do método Roadmapping, por exemplo, em consultorias com definição de escopo fechado feita a priori, isso poderia gerar ao menos dois prejuízos consideráveis: 1) litígio oriundo da demanda por modificar o escopo durante a aplicação; 2) baixa percepção de valor dos stakeholders durante a aplicação do método, devido a uma etapa que poderia ter sido sumarizada na etapa de planejamento da aplicação.

Uma solução pode ser encontrada no uso de lógicas contingenciais de uso do método. Lógicas que, a partir de características do ambiente, projeto, equipe e suas necessidades expressas especialmente durante a etapa de planejamento, pudesse orientar a processos mais ou menos detalhados de Roadmapping. Dito de modo simples: modelos que auxiliassem na compreensão - a priori - de particularidades que permitissem customizar mais intensamente a abordagem, priorizando o que é útil e reduzindo algumas partes do escopo de aplicação, tornando o processo de Roadmapping mais simples - sem perder a qualidade - como no caso aqui descrito. Ou, ainda, mais complexo e robusto - sem incorrer em prejuízos de tempo e recursos alocados.

Ainda, tal esforço em torno de uma lógica contingencial poderia contribuir para o desafio de definir corretamente o escopo de aplicação do método - ainda presente na literatura e prática do método (KERR; PHAAL, 2019). Exemplos de estudos que exploraram essa lógica 
contingencial, em maior ou menor nível, podem ser utilizados como inspiração, sendo encontrados no contexto dos processos de inovação (SALERNO et al, 2015), da gestão de projetos (BIANCHI; CONFORTO; AMARAL, 2021) e de processos de criação de startups (SOUZA; MELO FILHO; CHENG, 2020), embora ainda não sejam encontrados - dada a busca e o conhecimento dos pesquisadores deste artigo durante sua escrita - na literatura de Roadmapping.

\section{CONCLUSÃO}

O objetivo deste estudo foi aplicar o método Roadmapping, em formato digital, em um departamento de uma universidade, para a elaboração de um planejamento estratégico eficiente que possa condizer com os objetivos dos coordenadores do departamento.

A utilização de ferramentas digitais e métodos de tratamento de dados apoiaram todo o processo de aplicação do Roadmapping, evidenciando sua característica flexível. Assim, a construção do Roadmap foi eficiente e compreensível, o que fez com que seu aspecto visual fosse visto de uma forma mais simples e organizada, favorecendo para o entendimento de todos os participantes do processo e consumindo menos recursos do que a previsão inicial, que se baseava no modelo original de aplicação do Roadmapping.

A pesquisa para a busca e tratamento de dados foi útil para o início da aplicação, possibilitando uma visão rica e ampla sobre as oportunidades e ameaças do mercado. No entanto, elas poderiam ser reduzidas e priorizadas quando fossem necessárias, tendo em vista que a visão estratégica dos stakeholders já estava delimitada. Assim, o objetivo da aplicação foi resumido, e sem prejuízo percebido pelos stakeholders, em entender as ideias dos interessados e traduzi-las em um caminho comum.

Finalmente, a partir deste estudo foi feita uma proposta de estudo futuro que explorasse lógicas ou modelos contingenciais de apoio à customização do Roadmapping durante a etapa de planejamento, em termos de variáveis como características do ambiente, natureza e demandas do projeto, características e experiência da equipe envolvida e suas necessidades expressas. Tal proposta poderia robustecer a literatura e prática voltada ao Roadmapping, inspirando-se em estudos semelhantes realizados em outras áreas no contexto da Inovação e Tecnologia (ex.: BIANCHI; CONFORTO; AMARAL, 2021; SALERNO et al, 2015; SOUZA; MELO FILHO; CHENG, 2020). 


\section{REFERÊECIAS}

BIANCHI, Michael Jordan; CONFORTO, Edivandro Carlos; AMARAL, Daniel Capaldo. Beyond the agile methods: a diagnostic tool to support the development of hybrid models. International Journal of Managing Projects in Business, 2021.

KERR, Clive; PHAAL, Robert. Defining the scope of a roadmapping initiative: A checklistbased template for organizational stakeholders. In: 2019 Portland International Conference on Management of Engineering and Technology (PICMET). IEEE, p. 1-10, 2019.

SOUZA, Matheus L. P. et al. Agile Roadmapping: A management Tool for Digital Entrepreneurship. IEEE Transactions on Engineering Management, IEEE, p. 1-15, 2020.

SOUZA, M.; CANEDO, W.; BAGNO, R.. Roadmapping Ágil In: BAGNO, R.; SOUZA, M.; CHENG, L. (Eds.). Perspectivas sobre o empreendedorismo tecnológico: Da ação empreendedora aos programas de apoio e dinâmica do ecossistema. Curitiba: Brazil Publishing, 2020, p. 193-224.

OLIVEIRA, Maicon et al. Roadmapping: uma abordagem estratégica para o gerenciamento da inovação em produtos, serviços e tecnologias. Alta Books, 2019.

SALERNO, Mario Sergio et al. Innovation processes: Which process for which project?. Technovation, v. 35, p. 59-70, 2015.

PHAAL, R.; KERR, C. Technology roadmapping: Industrial roots, forgotten history and unknown origins. Technological Forecasting and Social Change, v.155, 2020.

PHAAL, R.; FARRUKH, C.J.P.; PROBERT, D.R. Strategic Roadmapping: A WorkshopBased Approach for Identifying and Exploring Strategic Issues and Opportunities. Engineering Management Journal, v. 19, n. 1, p. 3-12, 2007. 
PHAAL, R.; FARRUKH, C.; PROBERT, D. Roadmapping for Strategy and Innovation: Aligning technology and markets in a dynamic world. University of Cambridge, UK: Institute of Manufacturing. 2010.

PHAAL, R.; FARRUKH, C.J.P.; PROBERT, D.R. Technology Roadmapping: a Planning Framework for Evolution and Revolution. Technological Forecasting and Social Change, v.71, n. 1-2, p. 5-26, 2004.

PHAAL, R; FARRUKH, C; PROBERT, D. T-Plan: the fast-start to technology roadmapping: planning your route to success. University of Cambridge, Institute for Manufacturing, 2001.

YIN, R.K. Estudo de caso: Planejamento e Métodos. Editora Bookman, 2001. 